\title{
Three-dimensional Human Airway Segmentation Methods for Clinical Virtual Bronchoscopy ${ }^{\mathbf{1}}$
}

\author{
Atilla P. Kiraly, MS, William E. Higgins, PhD, Geoffrey McLennan, MD, PhD
}

Eric A. Hoffman, PhD, Joseph M. Reinhardt, PhD

Rationale and Objectives. The segmentation of airways from CT images is a critical first step for numerous virtual bronchoscopic (VB) applications. Automatic or semiautomatic methods are necessary, since manual segmentation is prohibitively time consuming. The methods must be robust and operate within a reasonable time frame to be useful for clinical VB use. The authors developed an integrated airway segmentation system and demonstrated its effectiveness on a series of human images.

Materials and Methods. The authors' airway segmentation system draws on two segmentation algorithms: (a) an adaptive region-growing algorithm and $(b)$ a new hybrid algorithm that uses both region growing and mathematical morphology. Images from an ongoing VB study were segmented by means of both the adaptive region-growing and the new hybrid methods. The segmentation volume, branch number estimate, and segmentation quality were determined for each case.

Results. The results demonstrate the need for an integrated segmentation system, since no single method is superior for all clinically relevant cases. The region-growing algorithm is the fastest and provides acceptable segmentations for most VB applications, but the hybrid method provides superior airway edge localization, making it better suited for quantitative applications. In addition, the authors show that prefiltering the image data before airway segmentation increases the robustness of both regiongrowing and hybrid methods.

Conclusion. The combination of these two algorithms with the prefiltering options allowed the successful segmentation of all test images. The times required for all segmentations were acceptable, and the results were suitable for the authors' VB application needs.

Key Words. Bronchi, CT; bronchoscopy; computed tomography (CT), image processing; computed tomography (CT), threedimensional; trachea, CT.

๑ AUR, 2002

New multidetector spiral CT scanners can produce threedimensional (3D) volumetric images of the human airway tree consisting of hundreds of two-dimensional (2D) sec-

\section{Acad Radiol 2002; 9:1153-1168}

${ }^{1}$ From the Departments of Computer Science and Engineering (A.P.K., W.E.H.), and Electrical Engineering (W.E.H.), Penn State University, University Park, Pa; and Departments of Radiology (W.E.H., E.A.H.), Internal Medicine (G.M.), and Biomedical Engineering (E.A.H., J.M.R.), University of lowa, 1402 SC, lowa City, IA 52242. Received June 10, 2002; revision requested June 19; revision received and accepted July 9 . Supported in part by grants CA74325 and CA91534 from the National Cancer Institute, by National Science Foundation grant 0092758, by a Biomedical Engineering Research Grant from the Whitaker Foundation, and by a National Institutes of Health Bioengineering Research Partnership (NIH-HL-064368). Address correspondence to J.M.R.

(c) AUR, 2002 tions $(1,2)$. A typical 3D image can include 400 or more $512 \times 512$ 0.6-mm sections. Such images provide an excellent basis for virtual bronchoscopy (VB) applications (3-15) and quantitative airway analysis (8,16-20). New VB methods also allow for live guided nodule and lymph node biopsies $(13,15)$. A critical first step in these VB applications is the segmentation of the airway tree. Manual interactive segmentation has been applied in some cases, but routine manual analysis is impractical for the large 3D images arising from the new scanners $(21,22)$. A variety of semiautomatic airway segmentation techniques have been proposed, but none have been conclusively proved adequate for very large, high-resolution, 3D CT chest images (4,20-30). 
We present an integrated airway segmentation system that includes both region-growing and mathematical morphology segmentation algorithms. Our system includes a hybrid method (31) that gives results equivalent to a previously proposed morphology-only approach (22) but greatly reduces the time required for segmentation. The hybrid method is based on the results of both regiongrowing and morphology approaches. We present a comparison study of the underlying image processing algorithms in our segmentation system. This study reveals that a small amount of filtering applied before segmentation can greatly increase the robustness of all methods in the case of patient breathing artifacts, stent distortion, and partial volume artifacts. We will also illustrate the utility of 3D airway segmentation in VB-assisted peripheral nodule analysis and review the airway segmentation problem and previously proposed segmentation methods.

A 3D CT image of the chest, $I$, comprises a stack of $Z$ contiguous sections. Each section consists of $X_{\text {size }} \times Y_{\text {size }}$ voxels (typically $512 \times 512)$. Each voxel $(x, y, z)$ has intensity value $I(x, y, z)$ and spacing $(\Delta x, \Delta y, \Delta z)$. The human airway tree appears on a 3D image as a set of connected, dark, branching tubular structures that tend to decrease in diameter as the branching progresses. CT images are reasonably well calibrated, such that air is at approximately $-1,000 \mathrm{HU}$ (ie, $I[x, y, z]=-1,000$ for a pure air voxel $[x, y, z])$ while water is at $0 \mathrm{HU}$. Soft tissues, such as those in the mediastinum, are situated in the range of -100 to $200 \mathrm{HU}$, and dense bone is near $1,000 \mathrm{HU}$.

Airway-tree segmentation is a challenging problem for several reasons. While airway voxels are generally near $-1,000 \mathrm{HU}$, noise and partial volume effects make it impossible to use a simple threshold to identify all airway voxels within an image (18). Whenever mixtures of different tissue types comprise a voxel, intermediate graylevel values are the result (32). Voxels straddling air and airway walls typically have values well above $-1,000$ HU. These partial voxels can be resolved into tissue components through statistical methods (32-35). Moreover, due to the size of the voxel, thin or stenosed airways can appear broken or discontinuous. Finally, image reconstruction artifacts, such as those introduced when a sharp high-frequency kernel is used, may cause the airways to appear discontinuous (36). Such discontinuities may cause problems during the segmentation, resulting in both under- and oversegmentation errors.

Previously proposed airway segmentation methods have employed four strategies: (a) knowledge-based tech- niques $(21,23,37),(b)$ region growing $(4,24,25,30,38)$, (c) central-axis analysis $(20,27)$, and $(d)$ mathematical morphology $(22,26,28,29)$. The technique proposed by Sonka et al $(21,23)$ uses an anatomic knowledge base describing structural relationships between airways and neighboring pulmonary vessels. Initially, 3D seeded region growing is used to identify large airways. The knowledge-based rules are applied to the image on a section-by-section basis. A fuzzy logic approach was later added by Park et al (37) to improve specificity. Neither method was tested on human data. More robust (less knowledge-dependent) methods exist.

Region-growing-based methods use voxel connectivity and a threshold to identify regions, usually through 26connectivity $(4,24,25,30,38)$. Summers et al (4) use 3D seeded region growing and a manually selected threshold to segment airways for rendering. Schlathölter et al (38) also use a set threshold in addition to other parameters to perform a type of 3D seeded region growing. Regiongrowing fronts are checked to prevent parenchymal leakage. The algorithm proposed by Mori et al (25) uses an adaptive 3D region-growing algorithm that automatically determines a threshold through repeated segmentations. Wood et al (24) also employ 26-connected region growing in conjunction with a global threshold as a basis for airway segmentation. Law and Heng (30) use adaptive 3D region growing but add a genetic algorithm to identify the center of the trachea automatically.

Although 3D region growing is extremely fast, it suffers from partial volume effects and noise due to the global threshold used during segmentation. The "optimal" thresholds differ for large versus small airways because of these factors. The resultant segmentation tends to lack finer details of the airways and contains rough edges. All of these similar methods can either lose details, depict incomplete structures, or suffer from parenchymal leakage ("explosion") to varying degrees.

Segmentation algorithms based on central axis analysis depend on central axis estimates for computing the segmentation $(20,27)$. The segmentation technique proposed by Swift et al $(20,27)$ performs a central axis computation as a basis for airway segmentation. This computation begins with a manually selected root site. The surface of an ellipsoid positioned at this site is then sampled to determine more sites or to detect the end of a branch. The process is then repeated for each new site. Further processing produces a complete central axis tree. Bronchial walls are then detected along this tree via thresholding. Finally, the unified results of this process determine the segmented 


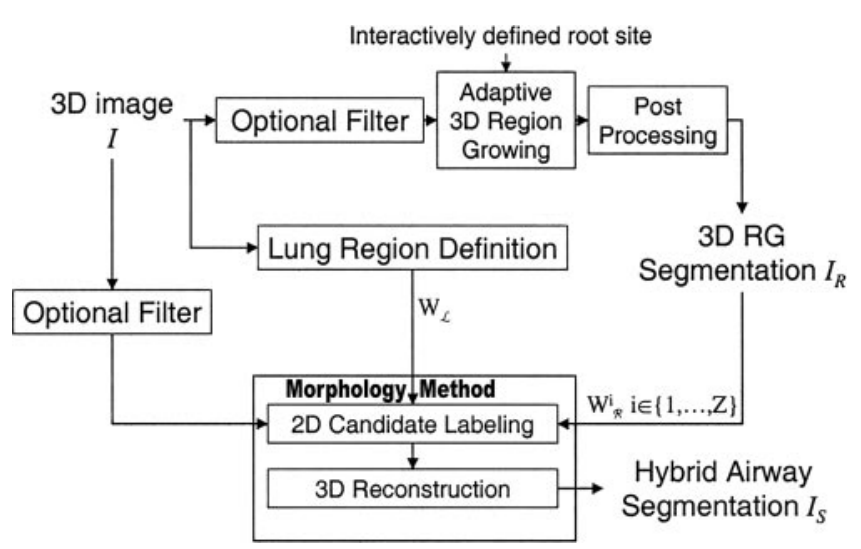

Figure 1. System diagram of the hybrid and region-growing airway segmentation algorithms.

airway tree. The disadvantage of this method is the critical dependence on the central axis analysis results, which may be imperfect or fail for several reasons. Concerns include complex dependencies of initial parameters and stopping criteria and the possibility of forming paths outside the airways.

The field of mathematical morphology involves imageprocessing operations that focus on shape and gray-scale properties $(39,40)$. Airway segmentation methods that draw on mathematical morphology tend to have two or more processing phases $(22,26,28,29)$. First, candidate airways are detected by means of various morphologic operations. Next, 3D relationships and shape characteristics help determine the true airways from false candidates. Bilgen et al (22) proposed an algorithm based on binary and gray-scale morphologic operations. Two-dimensional operators of varying sizes are applied to each section of the image to identify candidate airways. Next, false candidates are eliminated through 3D reconstruction. Pisupati et al (26) proposed a similar technique that uses the same principles to detect both pulmonary arteries and veins. Prêteux et al (28) use a method based on a combination of morphologic filtering, connection cost-based marking, and conditional watershed techniques to segment the bronchi of sheep lungs. Three-dimensional tree reconstruction was not considered, since the primary focus was 2D airway detection.

Extending this work to 3D airway segmentation and reconstruction, Fetita and Prêteux (29) have proposed methods that use more complex morphologic operations in addition to fractal analysis of candidate regions. An updated selective marking and depth-constrained connection cost operator is used to identify possible airways.
Valid candidates are identified through a 3D reconstruction procedure involving model-based aggregation and fractal analysis. While these methods are promising, their computation time tends to be inordinate, particularly for real clinical scenarios. Variations in the properties of the morphologic operators directly affect the candidates determined. In addition, the reconstruction process, once candidates are defined, can be complex and unestablished. Further, these methods have received little or no testing on large high-resolution 3D CT chest volumes.

We present a segmentation system that includes adaptive $3 \mathrm{D}$ region growing and mathematical morphology to give fast, robust methods for 3D airway-tree segmentation for chest CT images.

This article describes our airway segmentation system, presents results obtained by applying our system to human CT data, compares these results with other methods, and demonstrates virtual bronchoscopic (VB) analysis for bronchoscopic planning in a peripheral nodule case.

\section{MATERIALS AND METHODS}

This section describes our integrated 3D airway segmentation system, which allows the user to choose from three segmentation methods: (a) adaptive 3D region growing, $(b)$ morphologic based segmentation, and $(c)$ the hybrid method, which combines features of the other two methods. Figure 1 shows a layout of these component algorithms and how they comprise the system. Adaptive lung region definition and region growing are described first. We will then describe the other two methods and the image acquisition protocol for our test images.

\section{Lung Region Definition}

The lungs are identified on the 3D CT image with a simplified version of the method of $\mathrm{Hu}$ et al (41). In that method, lung segmentation is performed in three steps to produce separated left and right lungs with smooth boundaries. We used a simplified algorithm since we did not need precise results but only a bounding region for the lungs.

Lung region definition begins with the determination of a threshold to use for region growing at the root site. This threshold is large enough that the $3 \mathrm{D}$ region growing encompasses the entire lungs. The initial threshold, $T_{0}$, is set to $-1,000 \mathrm{HU}$. An iterative procedure then determines the final threshold. The segmentation threshold at step $i$, $T_{i}$, is used to separate the entire image into voxels greater 
than $T_{i}$ and those less than or equal to $T_{i}$. The new threshold, $T_{i+1}$, is set to

$$
T_{i+1}=\frac{\mu_{b}+\mu_{n}}{2},
$$

where $\mu_{b}$ and $\mu_{n}$ represents the mean gray level of the voxels greater than $T_{i}$ and that of all other voxels, respectively. This procedure is repeated until the condition $T_{i}=$ $T_{i+1}$ is met. The lung region is then identified by means of $3 \mathrm{D}$ region growing from the root site with threshold $T_{i}$.

\section{Adaptive Region Growing}

A robust adaptive 3D region-growing method provides an airway segmentation option in addition to identifying regions of the image containing the large airways for the hybrid method. We increased the robustness of the method described in reference 25 to provide good results for a wide variety of images. This section first describes adaptive 3D region growing and then describes our modifications to improve robustness.

Three-dimensional region growing uses a threshold $T$ and a root site $\left(x_{r}, y_{r}, z_{r}\right)$ to determine which voxels are added to the segmentation. In adaptive region growing, an optimal $T$ is found by repeating $3 \mathrm{D}$ region growing with increasing values of $T$. For airway segmentation, the threshold eventually becomes high enough that the region growing breaks through the bronchial wall and enters the lung parenchyma. The total volume $V$ of the segmentation is computed each time the $3 \mathrm{D}$ region growing is performed, so the breakthrough can be detected by means of a sharp increase in the volume, or explosion, and determined by a preset value called the explosion parameter, $E$, set to a value below the expected volume after explosion.

Let $I$ be the original gray-scale image, and let $I_{R}(x, y$, $z)=0, \forall(x, y, z) \in I_{R} . I_{R}$ will contain the output of the 3D region-growing segmentation. Let $N_{26}(x, y, z)$ be the 26-connected neighborhood of the voxel $(x, y, z)$, and let $T$ be the region-growing threshold. Given the root site, $\left(x_{r}, y_{r}, z_{r}\right)$, each neighbor $(x, y, z) \in N_{26}\left(x_{r}, y_{r}, z_{r}\right)$ such that $I(x, y, z)<T$ and $I_{R}(x, y, z) \neq 1$ is added to the segmentation; that is, we set $I_{R}(x, y, z)=1$. As each voxel is added, its neighbors are also processed in this fashion. This procedure is repeated until no new voxels are added to $I_{R}$. Then the volume $V$ of this segmentation is computed. The following steps outline how repeated 3D region-growing operations are used to determine an optimal

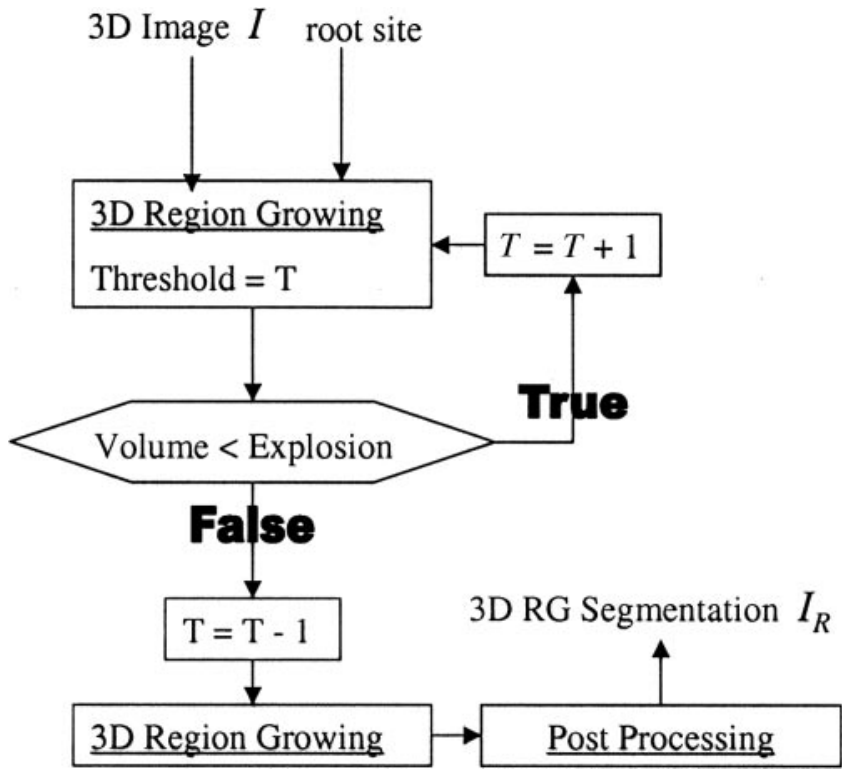

Figure 2. Block diagram of adaptive 3D region growing.

threshold: (a) initialize threshold to $T=-1,000 \mathrm{HU}$; (b) perform 3D region growing with root site $\left(x_{r}, y_{r}, z_{r}\right)$ and threshold $T ;(c)$ calculate the volume of region growing, $V$, from step 2, ie, $V=\delta x \times \delta y \times \delta z \times\left[\Sigma_{\forall(x, y, z)} \in\right.$ $\left.I_{R} I_{R}(x, y, z)\right] ;(d)$ if $V \leq E$, continue from step 2 with $T=T+1$; otherwise, $(e)$ decrease the threshold, $T=$ $T-1$, and perform final 3D region growing with this value. Figure 2 is a block diagram of these steps.

We have made two modifications to the adaptive 3D region-growing algorithm to increase robustness. The first modification is applied to the output segmentation and is represented by the "postprocessing" box in Figures 1 and 2. The final output of adaptive $3 \mathrm{D}$ region growing is unacceptable in most cases because of cavities in the segmentation due to noise in the original image. Additionally, the region boundaries in the final segmentation are often rough. We solve these problems by applying a $2 \mathrm{D}$ cavity filling and a 2D binary closing by a four-connected neighborhood operator to each section of $I_{R}(40)$.

The second modification is the prefiltering of the data. This option is manually applied as needed on a case-bycase basis. Prefiltering is necessary when even the minimal initial threshold creates an explosion into the parenchyma. Applying a 2D median filter to each section solves this problem (40). Two mask sizes are used, depending on the severity of the explosion. The first mask is a four-connected neighborhood and the second is a $3 \times$ 3 mask. The effects of these filters on region growing are described in Results. 


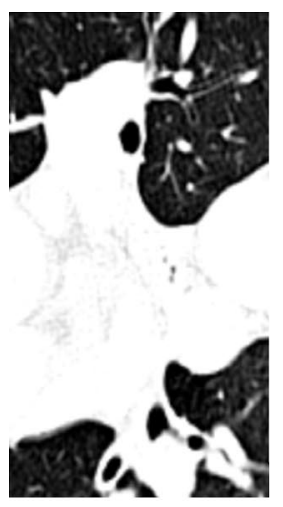

a.
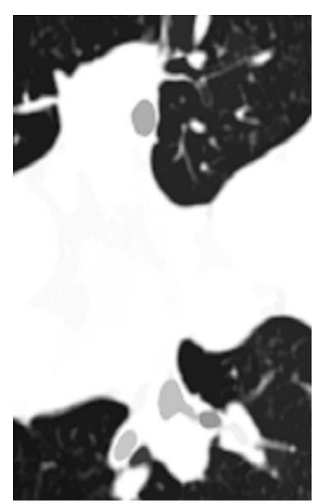

b.

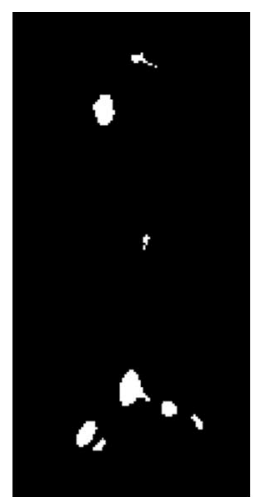

c.

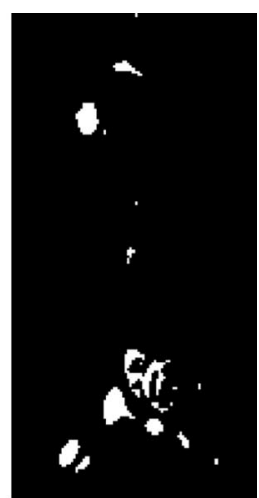

d.

Figure 3. Demonstration of $2 \mathrm{D}$ candidate labeling for case h016, section 283. (a) Original section is windowed to $(-1,000,0)$. (b) Gray-scale reconstruction is performed on the image in a with a operator of a specific size ( $J_{\infty}^{b}$ for a specific $b=7$ ). (c) Images $\mathbf{b}$ and $\mathbf{a}$ are subtracted and then thresholded by a value $T_{M}$ to produce a binary image of the candidate airways. (d) The union of binary images acquired through each operator is taken to define all candidate airways.

\section{Morphology-based Segmentation}

In the hybrid method, a modified form of the morphologic segmentation method proposed by Bilgen et al (22) accounts for most of the processing. The method of Bilgen et al has two steps. First, 2D morphologic operations are performed to determine candidate airway locations. This step uses gray-scale reconstruction followed by thresholding applied to each section. Next, 3D reconstruction is performed to recover the airway tree from these candidate locations. These steps are depicted in Figure 1. The result is a binary segmented volume of the airways, $I_{S}$.

Candidate airway locations are determined on a 2D basis by means of gray-scale reconstruction followed by thresholding. The basis operator for the reconstruction is given by a binary four-connected neighborhood, $B_{4}$, the smallest element used in the process. Larger elements are computed with repeated dilations of $B_{4}$ as follows:

$$
B_{4}^{b}=b B_{4}=B_{4} \oplus B_{4} \oplus \cdots \oplus B_{4}
$$$$
(b-1) \text { dilations }
$$

where $b B_{4}$ is the $b$ th-order homothetic of $B_{4}$.

An operator $B_{4}^{b}$ is applied to each individual section as described below. Given the original image $I$, each section $z=1, \ldots, Z$, of $I$ is windowed $(-1,000,0)$ to form a new 2D image $S$,

$$
S(x, y)= \begin{cases}I(x, y, z) & \text { if } I(x, y, z) \leq 0 \\ 0 & \text { otherwise }\end{cases}
$$

Windowing is used to eliminate the effect of variations in more dense structures, such as bone. Figure 3a shows an example of a windowed section. A marker image for gray-scale reconstruction, $J_{1}$, is then obtained from the gray-scale closing of $S$ with structuring element $B_{4}^{b}$,

$$
J_{1}^{b}=S \cdot B_{4}^{b}=\left(S \oplus B_{4}^{b}\right) \ominus B_{4}^{b}
$$

Hence, $J_{1}^{b}$ is computed from the gray-scale dilation by means of $B_{4}^{b}$ followed by an erosion by the same operator. Next, $J_{2}^{b}$ is computed from $J_{1}^{b}$ with the following:

$$
J_{k+1}^{b}=\max \left(J_{k}^{b} \ominus B_{4}, S\right),
$$

where $\max (.,$.$) computes the voxel-by-voxel gray-level$ maximum. Equation (3) is repeated until no further changes occur; that is,

$$
J_{\infty}^{b}(x, y)=J_{k+1}^{b}(x, y)=J_{k}^{b}(x, y), \quad \forall(x, y) \in J_{k}^{b},
$$

where $J_{\infty}^{b}$ represents the final gray-scale reconstructed image with structuring element $B_{4}^{b}$. In this image, local minima smaller than $B_{4}^{b}$ in $S$ are filled in with a gray-level value proportional to the difference between the maximum and minimum gray levels computed within a $B_{4^{-}}^{b}$ sized neighborhood of the minima. An example image of $J_{\infty}^{b}$ is shown in Figure $3 b$.

A gray-scale difference image is then computed between $J_{\infty}^{b}$ and $S$ and thresholded:

$$
C^{b}(x, y)= \begin{cases}1, & \text { if } J_{\infty}^{b}(x, y)-S \geq T_{M} . \\ 0, & \text { otherwise. }\end{cases}
$$

The difference image is bright where local minima exist in the image $S$. We use a value of $20 \%$ of the difference 
Figure 4. Coronal projections of the candidate labeled image and the image after 3D reconstruction.

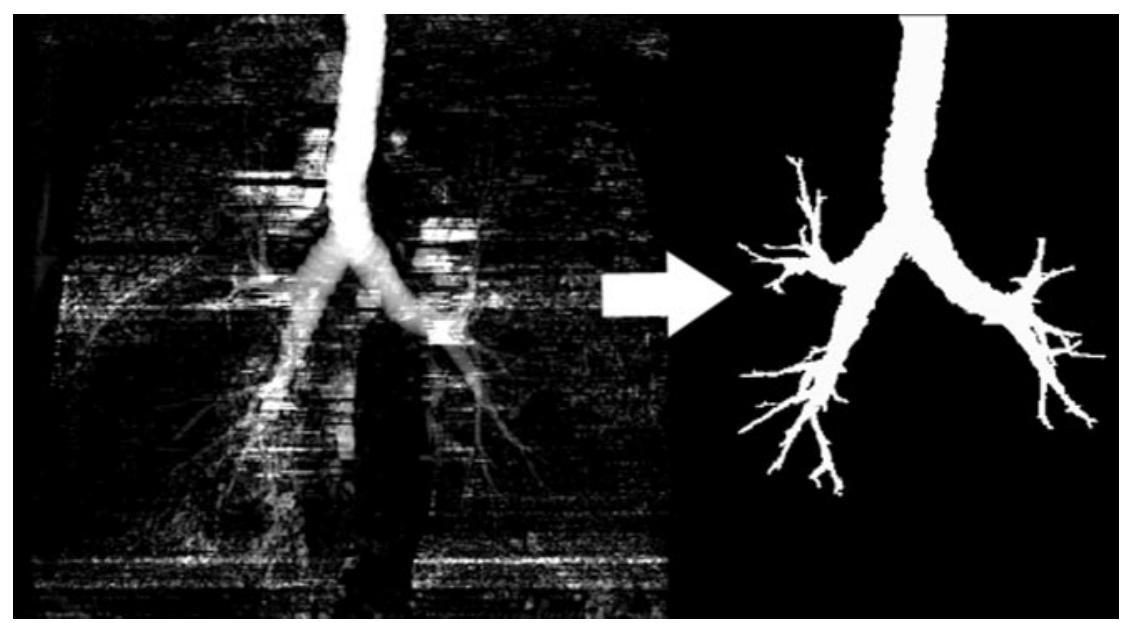

between the maximum and minimum possible values for the parameter $T_{M}$. In this case, since the data were windowed between 0 and $-1,000$, the threshold value is 200 . Figure $3 c$ gives an example of $C^{b}$, a binary section of potential airways identified by operator $B_{4}^{b}$.

To identify airways of various sizes, the above process is repeated for each element $B_{4}^{b}, b=1,2, \ldots, M$, where $B^{M}{ }_{4}$ is the maximum-sized operator. The union of this series of images is taken to form the final output $C$ for the section $z$ :

$$
C(x, y)=\bigcup_{b=1}^{M} C^{b}(x, y) .
$$

The segmented section $C$ contains all potential airways acquired through all the operators. An example of $C$ is shown in Figure $3 \mathrm{~d}$. $C$ is then inserted into $I_{S}$ to define candidate airway locations for section $z$ :

$$
I_{S}(x, y, z)=C(x, y), \quad \forall(x, y) \in C .
$$

This process is repeated for each section $z$ of $I$.

Once the candidate airway locations are in $I_{S}$, the $3 \mathrm{D}$ airway tree is reconstructed by using a closed space dilation with a unit kernel radius, as described in reference 22. Three-dimensional region growing with six-connectivity, rooted at the trachea, is used in this process, which eliminates most false candidates, leaving only the airways in $I_{S}$. An example is shown in Figure 4. Simpler reconstruction methods are also possible. A closing followed by region growing at the root site provides better true candidate recovery at the cost of greater false candidates.
An opening before this simpler reconstruction allows for better recovery of stenosed branches. In both of these operations, the operator used was a discrete sphere of radius 1 .

Segmentation methods using morphologic operations can be computationally expensive. Reference 22 describes the use of operators of various sizes to detect airways of corresponding sizes. These costly operators need not be applied to the entire image, however, but only to the approximate locations of the airways, because they have no theoretical relevance to other portions of the volume. The hybrid approach uses lung segmentation and adaptive 3D region growing to create masks that greatly constrain the volume where these operators are applied.

\section{Hybrid Lung Segmentation Algorithm}

A diagram of the hybrid algorithm is shown in Figure 1. The inputs are the 3D CT image of the chest and the location of the proximal end of the trachea, or the root site. The hybrid algorithm requires four parameters: (a) $E$, the explosion parameter for adaptive 3D region growing; (b) $s$, the maximal operator size considered "small"; (c) $M$, the maximal operator size for morphologic operations; and $(d) T_{M}$, the preset threshold used by the morphologic operator segmentation method. The final output is the segmented image of the airways, $I_{S}$.

The original image and root site are used throughout the method. The goal of the method is to provide the same quality of segmentation as an existing technique (22) but with an order of magnitude reduction in computation time. This increase in speed is achieved by using an adaptive 3D region-growing method (introduced in reference 25) combined with lung region definition. More 
specifically, we identify the locations of larger airways by using 3D region growing. Computationally expensive morphologic operators for large airways are then applied only to these identified regions. Areas of the lungs are masked by using lung region definition. Operators for smaller airways are applied in the restricted region encompassing only this area. These operators are characterized by their integer size, $b$. Several other segmentation approaches make use of morphologic operations followed by reconstruction $(26,28,29)$. These approaches can also be improved with our method.

Lung region definition creates a 3D mask of the data that defines the subvolume of the image containing the lungs. Once the lung region is segmented, the maximum bounding 3D parallelopiped of the segmentation, $W_{L}$, is used as a mask for smaller morphologic operators, $b=1$, $\ldots, s$. The Morphology-based Segmentation section describes how these operators of smaller sizes are applied on a section-by-section basis.

Adaptive 3D region growing uses an optional prefilter along with a specified explosion parameter, $E$, to provide a segmentation of the airways, $I_{R}$. The $E$ parameter simply ensures that an acceptable volume is segmented. This segmentation is used to provide a set of more refined masks for each section of the image, $W_{\mathrm{R}}^{i}, I=1, \ldots, Z$. These masks are then used by the remaining larger operators, $b=s+1, \ldots, M$. Although adaptive 3D region growing is fast, the segmentation does not always capture fine details on the order of one or two voxels, but it does give an excellent estimate of the location of larger airways. The computation time of this segmentation is small compared with that of any method employing morphologic operations. In summary, 3D region growing is used to create a rough segmented volume of the airways, $I_{R}$. For each section $I \in I_{R}$, a maximum bounding $2 \mathrm{D}$ region encompassing the segmented airways, $W_{R}^{i}$, is determined. Each window is then expanded by the radius of the largest operator to ensure proper detection of large airways on the boundaries.

Once the masks, $W_{L}$ and $W_{R}$, are computed, the reconstruction proceeds according the gray-scale reconstruction method described in Morphology-based Segmentation, but the operators are applied only to the regions dictated by these masks.

\section{Image Acquisition}

Thirty 3D CT images, obtained with either an MX8000 (Philips Medical Systems, Best, Netherlands) or an Aquilion (Toshiba America Medical Systems, Tustin, Calif) multisection CT scanner, were used to test the system. The subjects were studied under a protocol approved by the University of Iowa's Institutional Review Board. The scanning parameters for the Philips scanner included the following: $120 \mathrm{kVp}, 100 \mathrm{mAs}$ with a multisection pitch of 5 (equivalent to a single pitch of 1.25), 0.5-second rotation time, $0.6-\mathrm{mm}$ table increment, $180^{\circ}$ scan angle, and 1.3-mm section thickness. The images obtained with this scanner included four airway images, two lung nodule images, seven mediastinal lymph node images, and six other images, all obtained with a $D$ reconstruction kernel. In addition, eight images obtained with the MX8000 scanner were used to compare the A, B, and D reconstruction kernels. Three images from the Toshiba scanner were used to test the robustness of the system with a different scanner. All images were $512 \times 512$ pixels in the transverse plane but varied in resolution and the number of sections. Table 1 lists the testing images in detail.

\section{RESULTS}

In this section we present the results of the segmentation system applied to the test images. Various components of the system are compared, and the effects of filtering are presented. We then demonstrate the application of these methods to human VB analysis of a peripheral nodule. Adaptive region growing, morphologic reconstruction, and the hybrid method were compared for the images listed in Table 1. In each case, the root site was manually identified. Computation times were determined on a 933-MHz Xeon PIII personal computer (Dell Precision 620; Dell Computer, Austin, Tex) with two central processing units and 2 GB of memory. The explosion parameter, $E$, was set to $50,000 \mathrm{~mm}^{3}$ for all but one case. The threshold parameter for the morphologic method was kept at $T_{M}=200$ for all cases. The largest operator classified as small, $s$, was determined from the smallest airway $3 \mathrm{D}$ region growing can segment continually. A value of 3 was used for $s$ since airways with cross sections larger than $B^{3}{ }_{4}$ are hardly influenced by partial volume effects. The largest operator, $M$, is based on the size of the largest airway on the image. We found that setting $M=18$ adequately captures all the airways in our data sets.

The morphology and hybrid methods were first compared in terms of average execution time for 10 of the images in Table 1. In each case, the region-growing input to the hybrid method was checked to make sure that no major parenchymal leakage occurred. Minimal manual 
Table 1

Images Used in Testing the Algorithms Making up the 3D Airway Segmentation System

\begin{tabular}{|c|c|c|c|c|c|}
\hline Image & $Z$ & $\Delta x$ & $\Delta y$ & $\Delta z$ & Comments \\
\hline h017 & 239 & 0.56 & 0.56 & 0.50 & Toshiba \\
\hline h022 & 280 & 0.58 & 0.58 & 0.50 & Toshiba \\
\hline h023 & 100 & 0.60 & 0.60 & 3.00 & Toshiba \\
\hline h010 & 535 & 0.69 & 0.69 & 0.60 & Philips, D kernel \\
\hline h015 & 476 & 0.57 & 0.57 & 0.60 & Philips, D kernel \\
\hline h018frc & 448 & 0.57 & 0.57 & 0.60 & Philips, D kernel \\
\hline h018tlc & 475 & 0.57 & 0.57 & 0.60 & Philips, D kernel \\
\hline h001a & 414 & 0.57 & 0.57 & 0.60 & Philips, A kernel \\
\hline h008 & 389 & 0.59 & 0.59 & 0.60 & Philips, A kernel \\
\hline h026 & 502 & 0.76 & 0.76 & 0.60 & Philips, A kernel \\
\hline h026b & 502 & 0.76 & 0.76 & 0.60 & Philips, B kernel \\
\hline h001 & 414 & 0.57 & 0.57 & 0.60 & Philips, D kernel, lung nodule \\
\hline h019 & 597 & 0.74 & 0.74 & 0.60 & Philips, D kernel, lung nodule \\
\hline h003 & 210 & 0.66 & 0.66 & 0.60 & Philips, D kernel, airway case \\
\hline h009 & 492 & 0.69 & 0.69 & 0.60 & Philips, D kernel, airway case \\
\hline h012 & 149 & 0.62 & 0.62 & 0.60 & Philips, D kernel, airway case-papilloma \\
\hline h016 & 556 & 0.72 & 0.72 & 0.60 & Philips, D kernel, airway case \\
\hline h002 & 515 & 0.59 & 0.59 & 0.60 & Philips, D kernel, mediastinal lymph node \\
\hline h004 & 262 & 0.59 & 0.59 & 0.60 & Philips, D kernel, mediastinal lymph node \\
\hline h005 & 479 & 0.59 & 0.59 & 0.60 & Philips, D kernel, mediastinal lymph node \\
\hline h006 & 574 & 0.72 & 0.72 & 0.60 & Philips, D kernel, mediastinal lymph node \\
\hline h007 & 488 & 0.65 & 0.65 & 0.60 & Philips, D kernel, mediastinal lymph node \\
\hline h008 & 389 & 0.59 & 0.59 & 0.60 & Philips, D kernel, mediastinal lymph node \\
\hline h014 & 411 & 0.66 & 0.66 & 0.60 & Philips, D kernel, mediastinal lymph node \\
\hline h028Afrc & 370 & 0.68 & 0.68 & 0.60 & Philips, A kernel \\
\hline h028Bfrc & 370 & 0.68 & 0.68 & 0.60 & Philips, B kernel \\
\hline h028Dfrc & 370 & 0.68 & 0.68 & 0.60 & Philips, D kernel \\
\hline h029Afrc & 412 & 0.66 & 0.66 & 0.60 & Philips, A kernel \\
\hline h029Bfrc & 412 & 0.66 & 0.66 & 0.60 & Philips, B kernel \\
\hline h029Dfrc & 412 & 0.66 & 0.66 & 0.60 & Philips, D kernel \\
\hline
\end{tabular}

Note.- Images are listed by case number. $Z=$ number of sections, $\Delta x$ and $\Delta y=$ axial plane resolutions, and $\Delta z=$ section thickness. Comments indicate the scanner used (Philips MX8000 or Toshiba Aquilon), the reconstruction kernel, and case details (see Image Acquisition for additional information).

interaction was required to assure this condition. The hybrid method performed labeling more than 20 times faster on average (641 vs 13,751 seconds), and the $3 \mathrm{D}$ reconstruction time was also improved by $8 \%$ (737 vs 799 seconds).

Next, the adaptive region-growing and hybrid methods were compared. Two main factors were analyzed: robustness and execution time. Robustness was defined as the method's ability to complete a visually acceptable segmentation. Qualities such as segmentation volume and number of branches were also compared. The hybrid and region-growing methods were compared according to these criteria for all the images.

Table 2 shows the quality of segmentations according to the methods and filtering levels. It also divides the im- ages into those reconstructed with the $\mathrm{D}$ kernel and those reconstructed with the A and B kernels. All of the images were successfully segmented by both methods, although in some cases prefiltering was required for a successful segmentation. The quality of the segmentations was determined subjectively according to the quality of the branches segmented. Table 3 lists the mean segmentation times for both methods, with standard deviations and ranges. The average volumes were computed for all successful segmentations. There was a general decrease in volume with increasing filtering for both methods. This decrease was greater with the region-growing method. For the region-growing method, the average volumes dropped by approximately $20 \%$ and $21 \%$ for the star median and $3 \times 3$ median filters, respectively. The hybrid method 
Table 2

Results with the Hybrid and Region-growing Algorithms with Various Filters

\begin{tabular}{|c|c|c|c|c|}
\hline \multirow[b]{2}{*}{ Method and Filter } & \multicolumn{4}{|c|}{ No. of Images } \\
\hline & Excellent & $\begin{array}{l}\text { Minor } \\
\text { Branch } \\
\text { Fusion }\end{array}$ & $\begin{array}{l}\text { Major } \\
\text { Branch } \\
\text { Fusion }\end{array}$ & Failure \\
\hline \multicolumn{5}{|l|}{ Region-growing } \\
\hline No filter & 14 & 8 & 4 & 4 \\
\hline Star median & 21 & 3 & 5 & 1 \\
\hline $3 \times 3$ median & 23 & 6 & 1 & 0 \\
\hline \multicolumn{5}{|l|}{ Hybrid } \\
\hline No filter & 12 & 14 & 1 & 3 \\
\hline Star median & 17 & 8 & 2 & 3 \\
\hline $3 \times 3$ median & 17 & 9 & 1 & 3 \\
\hline \multicolumn{5}{|l|}{ Region-growing $A B$} \\
\hline No filter & 6 & 1 & 0 & 0 \\
\hline Star median & 6 & 1 & 0 & 0 \\
\hline $3 \times 3$ median & 7 & 0 & 0 & 0 \\
\hline \multicolumn{5}{|l|}{ Hybrid AB } \\
\hline No filter & 4 & 3 & 0 & 0 \\
\hline Star median & 5 & 1 & 1 & 0 \\
\hline $3 \times 3$ median & 6 & 1 & 0 & 0 \\
\hline \multicolumn{5}{|l|}{ Region-growing D } \\
\hline No filter & 8 & 7 & 4 & 4 \\
\hline Star median & 15 & 2 & 5 & 1 \\
\hline $3 \times 3$ median & 16 & 6 & 1 & 0 \\
\hline \multicolumn{5}{|l|}{ Hybrid D } \\
\hline No filter & 8 & 11 & 1 & 3 \\
\hline Star median & 11 & 7 & 1 & 3 \\
\hline $3 \times 3$ median & 10 & 8 & 1 & 3 \\
\hline
\end{tabular}

Note.-The quality of the segmentation was subjectively determined according to the fusing of branches. Images rated as excellent had no noticeable fusion. The next two categories included images with fusion of small peripheral branches and those with fusion of larger branches. Finally, failure was defined as large sections fused or incorrectly segmented images. The first six rows give results for all images, while the remaining rows give results for images reconstructed with the $A$ or $B$ and the $D$ kernel, respectively. For path planning for navigational purposes, all images are acceptable except for those considered failures. Figure $9 \mathrm{a}$ is an example of major branch fusion, and Figure 9b shows a segmentation classified as excellent. The segmentation on the left in Figure 7 a shows minor branch fusion.

showed average volume drops of $1.2 \%$ and $2.5 \%$ for the same filters and also demonstrated $4 \%$ greater average volumes for nonfiltered segmentations compared to the region-growing method. Finally, the number of branches of segmentations considered "excellent," as listed in Table 2 , were counted by an automatic path-planning algorithm (42). The region-growing segmentations had an average of 55 branches, while the hybrid method segmentations had an average of 74 branches.
Table 3

Timing Comparisons for Region-growing and Hybrid Methods

\begin{tabular}{lc}
\hline \multicolumn{1}{c}{ Method } & Mean Time $(\mathrm{sec})$ \\
\hline $\begin{array}{l}\text { Region growing } \\
\text { No filter }\end{array}$ & $19 \pm 12 \quad(5-67)$ \\
$\begin{array}{l}\text { Star median } \\
3 \times 3 \text { median } \\
\text { Hybrid }\end{array}$ & $70 \pm 18 \quad(17-92)$ \\
$\quad$ No filter & $116 \pm 29(28-158)$ \\
& \\
Star median & Lab: $761 \pm 357(198-1,561)$ \\
& Rec: $947 \pm 571(113-2,365)$ \\
$3 \times 3$ median & Lab: $745 \pm 332(184-1,478)$ \\
& Rec: $835 \pm 509(90-2,247)$ \\
& Lab: $730 \pm 303(176-1,383)$ \\
& Rec: $719 \pm 408(90-1,604)$ \\
\hline
\end{tabular}

Note.-Only successfully segmented images were considered in these statistics. Times are given as means \pm standard deviations, with ranges in parentheses. Lab $=$ labeling time, $\mathrm{Rec}=$ reconstruction time. The region-growing method is considerably faster than the hybrid method, but both are acceptable for clinical use.

Figures 5, 6, and 7a show the maximum-intensity projection views of airways segmented with the three methods. Figures $7 \mathrm{~b}$ and 8 show surface renderings based on the segmentation results.

Figure 5 shows case h007 segmented with the three different segmentation methods and three levels of filtering. The filtering enables segmentations to be completed successfully. All three methods displayed comparable segmentation quality, but the hybrid method showed no small explosion artifacts and achieved acceptable segmentation with less filtering than the region-growing method required. Figure 8 shows the surface renderings of the airways segmented with the $3 \times 3$ median filter.

Figure 7a shows the results in case h006, without filtering. The 3D region-growing and hybrid methods had similar results. Each method missed airways recovered by the other. With the region-growing method there was a minor explosion in the segmentation. Filtering or lowering the explosion parameter can correct this problem, but since the explosion volume is small, it would be difficult to determine the proper setting according to the volume change during region growing. Figure $7 \mathrm{~b}$ displays a surface-shaded view based on the hybrid segmentation results.

Figure 6 displays the results obtained without filtering in case h008. The hybrid method was the only successful one. Region growing failed because a low gray-level voxel directly linked the airways to the parenchyma. The mathematical morphology method failed because larger 


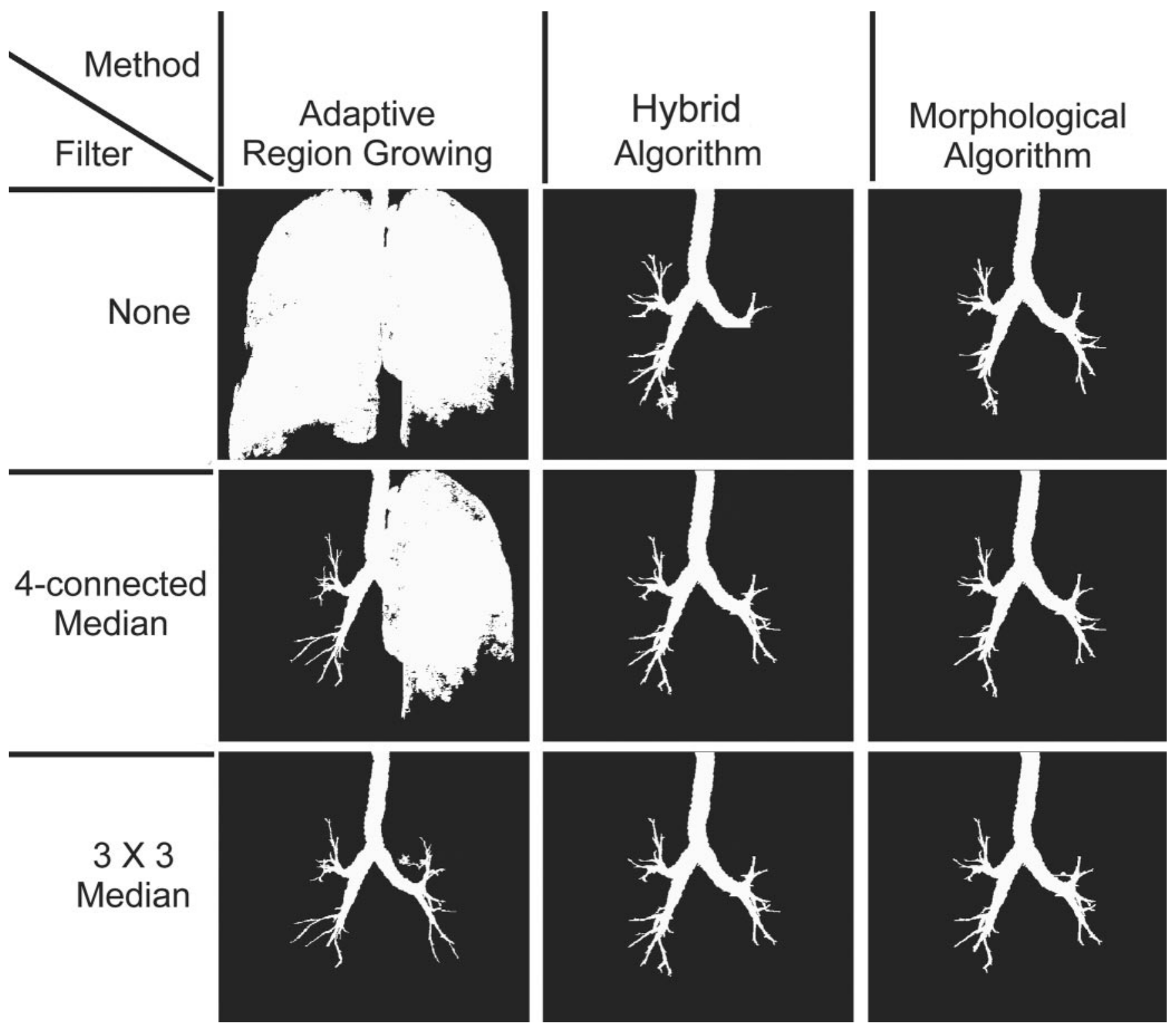

Figure 5. Maximum-intensity projection images of different segmentations of a $512 \times 512$ CT image of the chest (case h007) (488 sections; voxel spacing, $\Delta x=\Delta y=0.654297 \mathrm{~mm}, \Delta z=0.599976 \mathrm{~mm}$; image size, $244 \mathrm{MB}$. The three segmentation algorithms are shown in the columns, and the different levels of filters in the rows. Filtering the image before processing increases the robustness of the segmentation methods, but it also tends to remove smaller branches or identify nonairway regions, depending on the algorithm used (root site $\left.=[266,221,0], E=50,000 \mathrm{~mm}^{3}, T_{M}=200\right)$.

structuring elements identified candidates that eventually linked airways to the parenchyma. Filtering prevented failure in the latter two methods.

We have applied the segmentation method to over 30 human cases thus far, using an integrated VB system $(11,20,44,45)$. The system permits assessment of 3D CT images and live guidance of follow-up bronchoscopy. Reference 45 illustrates the system's use for phantom studies, while reference 20 describes preliminary efforts to assess 3D CT images in human subjects.
Figures 9 and 10 illustrate the use of our segmentation method and the VB system in a peripheral nodule case. The initial 3D CT image was obtained with a Philips MX8000 multidetector helical CT scanner and consisted of 414 sections $(512 \times 512$; section thickness, $0.6 \mathrm{~mm}$; axial plane resolution, $0.566 \mathrm{~mm}$ ). Figure 9 illustrates airway-tree segmentation results with and without filtering. After segmentation, we used an automated algorithm to compute the centerline paths for the segmented tree (42). Twelve generations of airway branches were ex- 


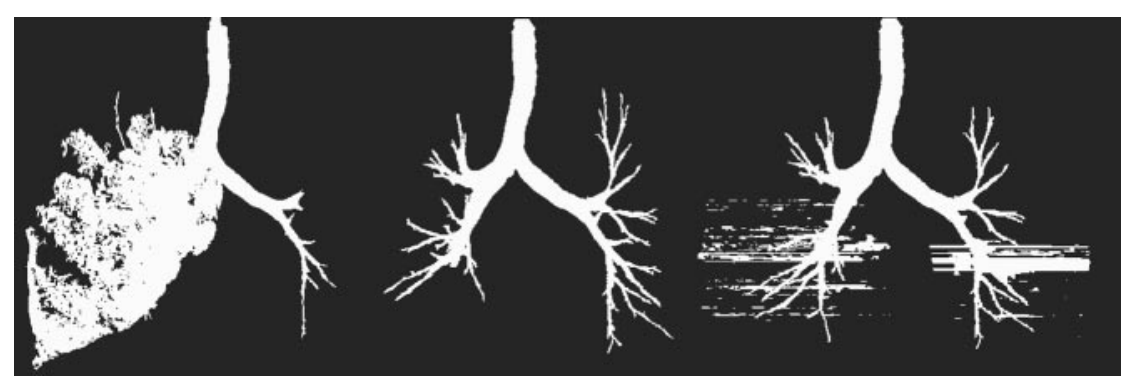

Figure 6. Maximum-intensity projections of three different segmentation algorithms for a $512 \times 512$ CT image of the chest (case h008) (389 sections; voxel spacing, $\Delta x=$ $\Delta y=0.585938 \mathrm{~mm}, \Delta z=0.599976 \mathrm{~mm}$; image size, $194 \mathrm{MB})$. The left image was obtained with adaptive $3 \mathrm{D}$ region growing, the center image with the hybrid algorithm, and the right image with the standard morphologic algorithm (failed). All three images were computed without filtering (root site $=[242,211,0], E=50,000 \mathrm{~mm}^{3}, T_{M}=200$ ).
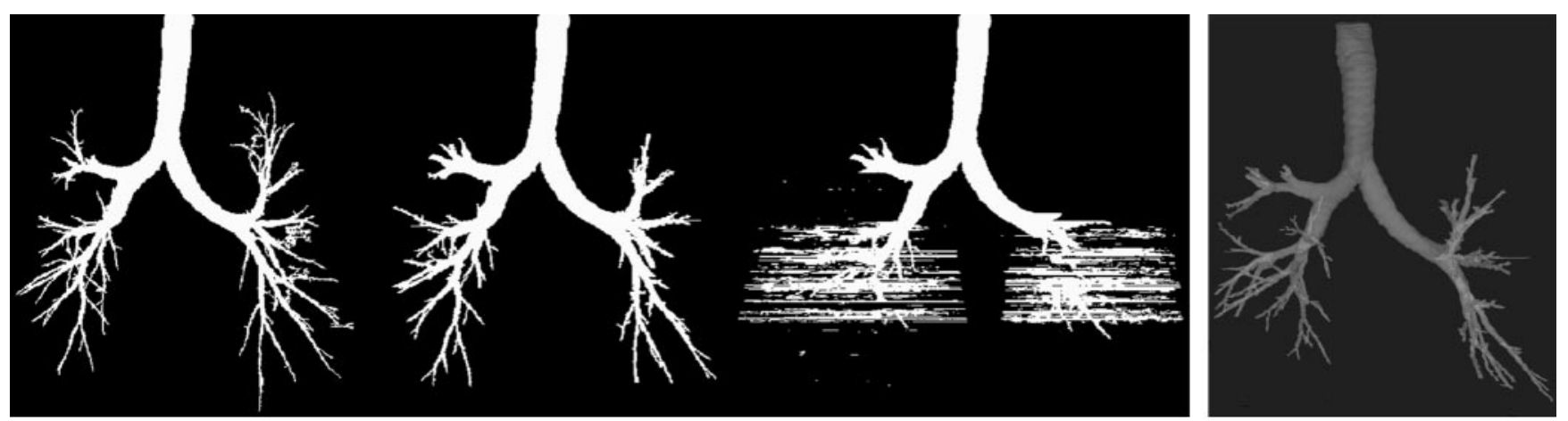

a.

b.

Figure 7. (a) Maximum-intensity projections of three different segmentation algorithms for a $512 \times 512$ CT image of the chest (case h006) (574 sections; voxel spacing, $\Delta x=\Delta y=0.724609 \mathrm{~mm}, \Delta z=0.599976 \mathrm{~mm}$; original image size, $287 \mathrm{MB}$ ). The left image was obtained with adaptive 3D region growing, the center image with the hybrid algorithm, and the right image with the standard morphologic algorithm. All three images were computed without filtering. The center image was used to mask the original gray-scale data. (b) The marching-cubes algorithm with a threshold of $-600 \mathrm{HU}$ was then applied to the masked gray-scale data to create the surface shown (root site $\left.=[273,248,0], E=50,000 \mathrm{~mm}^{3}, T_{M}=200\right)$.

tracted, which included 182 branches and 93 paths (see reference 20 for definitions); some paths toward the periphery are false. The segmentation step took 2 minutes 26 seconds, compared with 3 minutes 19 seconds for the path analysis. All processing was done on a Dell Inspiron (Dell Computer) 3800 laptop computer (700-MHz central processing unit, 512-MB RAM).

Figure 10 gives a composite VB system view for the case. A peripheral nodule is clearly visible on the coronal projection view in the right lung. We manually defined the nodule with the system's built-in editing tools. We then created a surface file by using a custom surface-dilation procedure and a standard marching cubes algorithm (46). All tools clearly show the nodule, while the endoluminal renderer shows an interior view in the right main bronchus. These data could be used for planning follow-up biopsy.

\section{DISCUSSION}

We have presented an airway segmentation system based on region-growing and mathematical morphology algorithms for segmentation. We have evaluated the underlying algorithms by testing the system with a group of 30 images. The results show that no single method is superior in all respects, demonstrating a need for a multifaceted segmentation system. The use of two methods along with filtering allows all the images to be successfully segmented. In this section we discuss the effects of filtering on segmentation robustness and quality, the different reconstruction kernels, and the results of the hybrid algorithm compared with the other methods.

Filtering the image before segmentation increases the robustness of the algorithms by helping eliminate lowdensity voxels that connect the airways to the paren- 


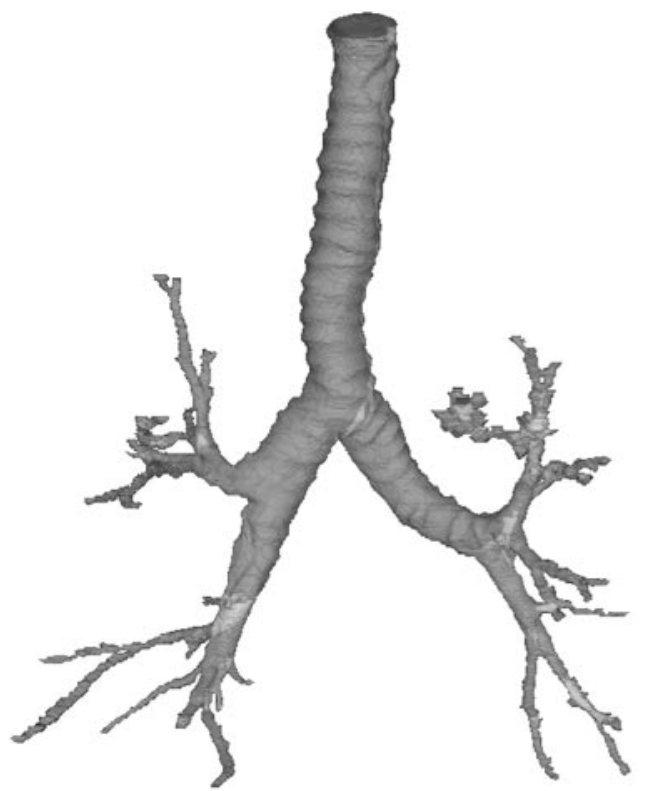

a.

Figure 8. Surface renderings based on the segmented images from Figure 5 obtained with the $3 \times 3$ median filter and (a) the regiongrowing, (b) hybrid, and (c) morphology methods. The segmentations were dilated by three voxels and used as a mask on the original gray-level data. The marching-cubes algorithm with a threshold of $-800 \mathrm{HU}$ was then used on the masked data (43).

Figure 9. Coronal maximum-intensity projection images of a segmented airway tree obtained with 3D CT of a peripheral nodule (see text for image details). (a) Image obtained with region growing and no filtering $\left(\right.$ root site $\left.=[273,292,0] ; E=50,000 \mathrm{~mm}^{3}\right)$. Note the extraneous noisy extensions. (b) Image obtained with the same segmentation parameters as in a but with a star median filter (five-point windows, including the central pixel and its four neighboring pixels along the vertical and horizontal axes); the view is markedly cleaner.
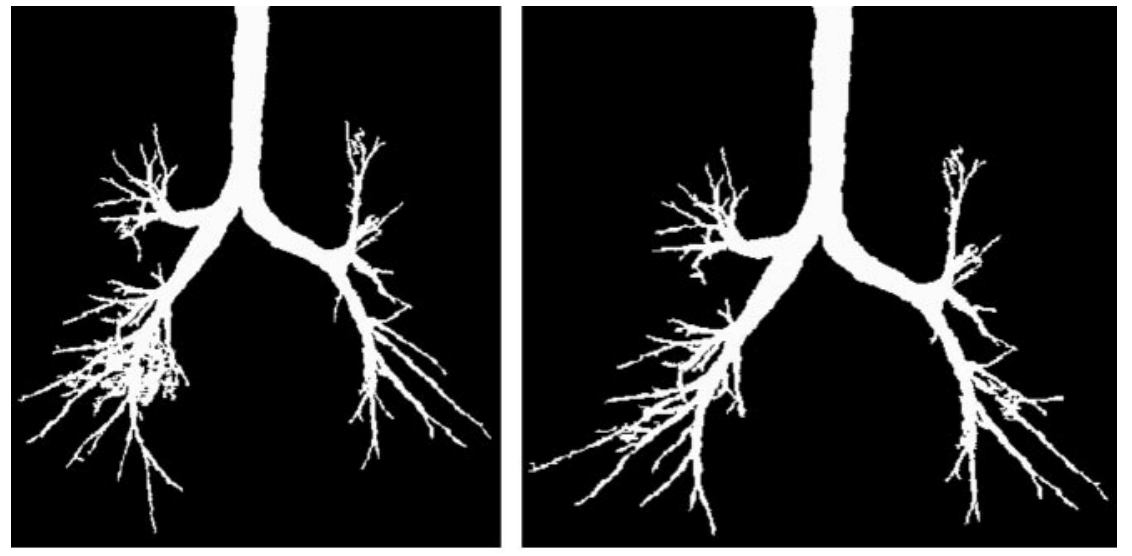

a.

b. chyma. In most of the cases shown in Table 2, the number of excellent segmentations increased with greater filtering.

Filtering has predictable effects on the region-growing segmentation. In the column for the region-growing method in Figure 5, there are two noticeable effects of increased filtering. The first is successful segmentation. The second effect is negative, in that smaller branches are eliminated. The hybrid and morphologic methods do not respond as predictably to filtering. This behavior is due to the more complex two-step process used in computing the segmentation. Filtering has a less predictable effect on the morphologic operations but can still result in improvements in some cases. A trend toward more segmentations classified as excellent is noticeable for both methods in Table 2 .

The hybrid method tends to be more robust against failure than the original morphology method or the region-growing method when filtering is not used. The use of filtering benefits the region-growing algorithm the most, since the hybrid algorithm is less prone to leaks into the parenchyma. Since filtering tends to reduce the 


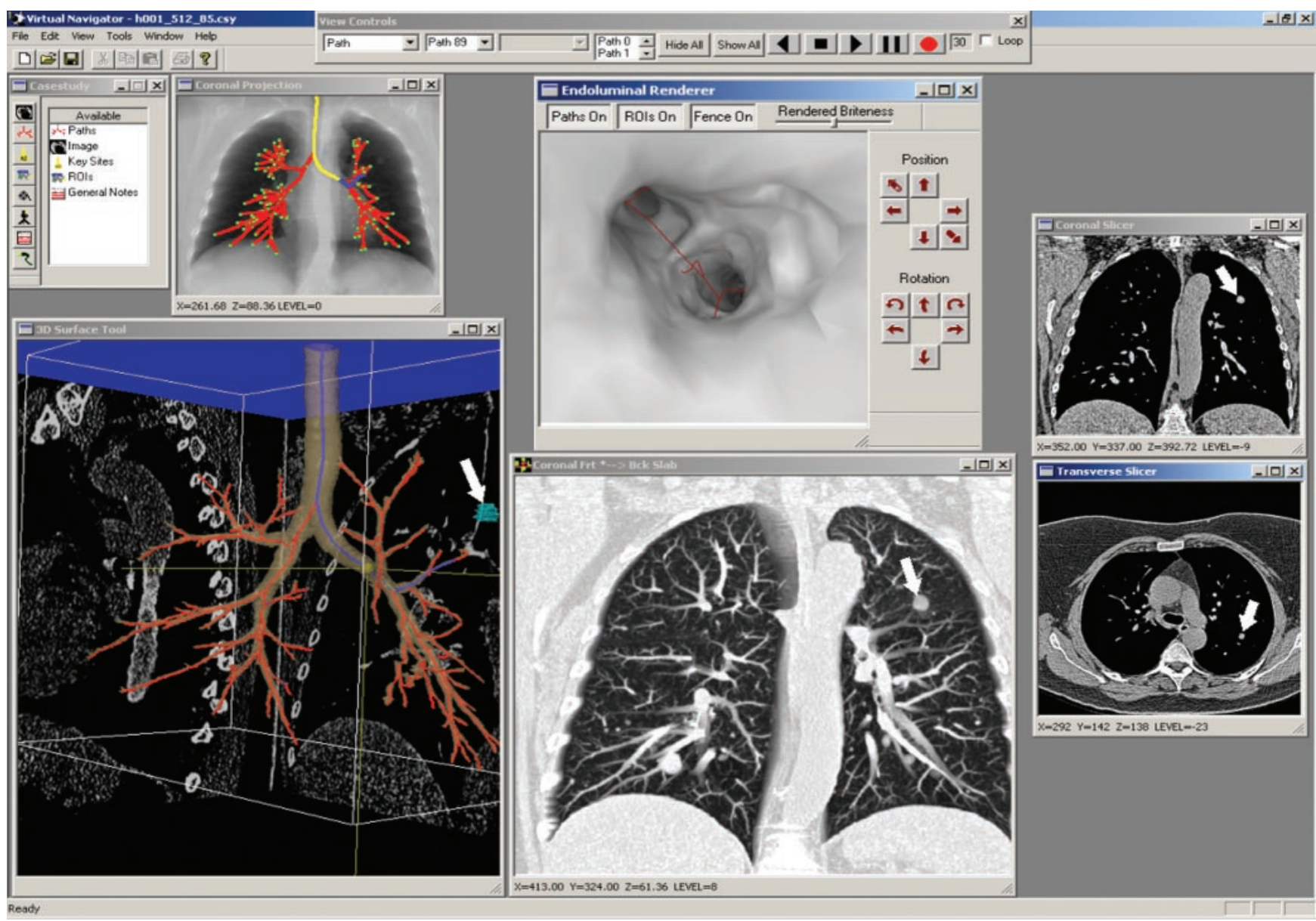

Figure 10. Composite view in a VB system for the peripheral nodule case shown in Figure 9. The 3D surface tool shows the rendered airway tree and precomputed airway centerlines. One path that leads closest to a peripheral nodule is highlighted. Arrows on the various tools indicate the location of the nodule. The coronal projection view is a half-sized maximum-intensity projection of all data between $Y=150$ and $Y=400$. It shows the nodule and the highlighted (white) path closest to it. The Coronal Front-to-Back Slab depicts a depth-weighted maximum view near the nodule (field of view $=30$, depth of vision $=40$, viewing window $=[-1,024,196])(47)$. The Transverse and Coronal Slicer views show original section data in the transverse and coronal directions; a mediastinal viewing window was used $(\mathrm{WL}=-40, \mathrm{WW}=400)$. Finally, the Endoluminal Renderer shows an interior airway view approaching the end of the right main bronchus; this site is shown by the ball on the 3D surface view and the termination of the white path on the coronal projection view (case h001) (root site $=[273,292,0]$, region growing, star median filter, $E=50,000 \mathrm{~mm}^{3}$, slab $=[$ focus $=20$, vision $=30$, maxwin $=$ 400]).

number of peripheral branches, the segmentation volume tends to decrease. The hybrid method, however, is less influenced by filtering.

In summary, filtering increases the robustness of these algorithms but comes at the cost of losing smaller branches or identifying additional nonairway structures. For several images, segmentation with adaptive region growing was unsuccessful, but prefiltering solved the problem in each case. The four-connected neighborhood median filter eliminates the finer branches, and the $3 \times 3$ median filter eliminates thicker ones.

The assortment of images included several different reconstruction kernels. Table 2 distinguishes images re- constructed with the D kernel and those reconstructed with the A or B kernel. The images are classified into four levels of quality, from excellent to failure. The ratio of excellent images to other quality levels is higher for images reconstructed with the A or B kernel. This property is seen for both reconstruction methods. The D kernel has more instances of branch fusion and failures. It offers a sharp reconstruction for more pronounced edges. This quality also produces more noise and strengthens artifacts, reducing the segmentation quality. Voxels that make up the airway walls in the peripheral airways are altered more with the D kernel, creating a fusion of peripheral branches. These results suggest that an A or B 
Figure 11. Sections of gray-scale 3D CT data with overlays of the resultant segmentations obtained with region-growing and hybrid methods. The hybrid method demonstrates better edge localization since it captures more of the airway lumen than the regiongrowing method. This property partly explains why the hybrid method results in greater segmentation volumes. This feature also makes hybrid segmentation better for quantitative analysis.
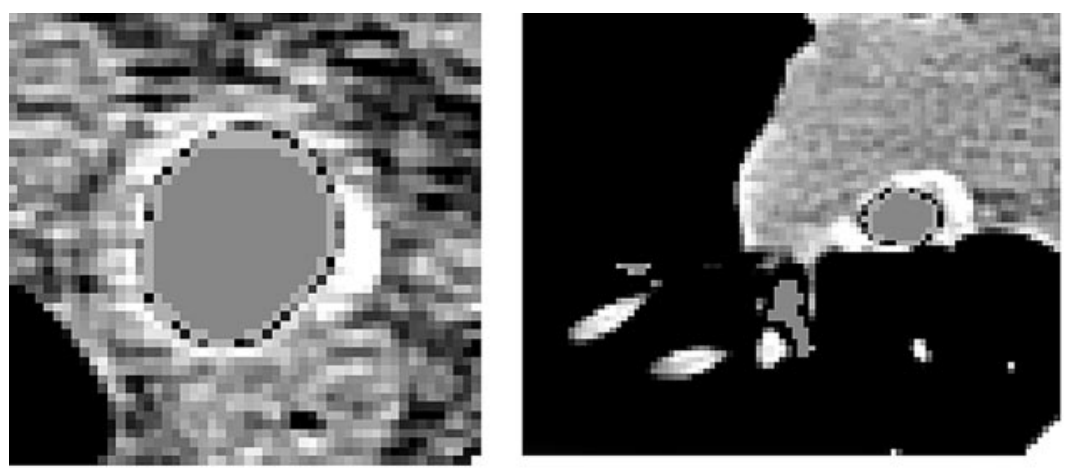

\section{Segmented by Hybrid method only}

\section{Segmented by both RG and Hybrid methods}

Figure 12. (a) Surface rendering of the airway from a patient with papilloma (case h012). Region growing was the only successful method. (b) Magnified view shows a windowed $(-1,000,0)$ section of the data. The morphology method does not segment the cavity successfully due to the cavity's odd size, shape, and contents. This case demonstrates the necessity of the region-growing method in a clinical environment (root site $=$ $[266,221,0]$, region growing, star median filter, $E=70,000 \mathrm{~mm}^{3}, T_{M}=200$ ).
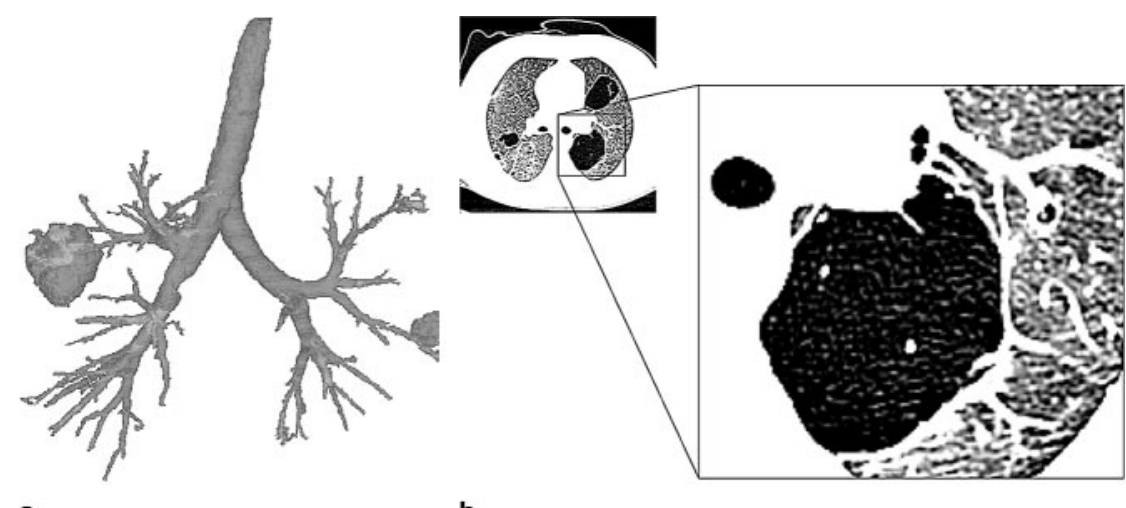

b. reconstruction kernel would be more appropriate for segmentation. Reinhardt et al (18) also found that a normal reconstruction kernel is better suited for quantitative purposes.

As previously mentioned, neither method is superior. The adaptive region-growing and hybrid methods each have distinct advantages. The hybrid method, however, is superior to the morphology method in that it substantially reduces execution times while producing very similar results. These conclusions are discussed below.

Adaptive $3 \mathrm{D}$ region growing proved to be the fastest method for obtaining an airway segmentation, but several airways can be missed and the lumen edge definition tends to be poor. Figure 11 demonstrates the superior edge localization of the hybrid method. Although fusion of the branches increases the segmented volume slightly and the hybrid tended to have more fusion cases, the edge localization is probably why its segmentation volume tended to be greater. Furthermore, for the images classified as excellent, those segmented with the hybrid method had more branches on average than those segmented with region growing: 74 versus 55 .

As one case demonstrates, however, adaptive region growing is a necessary method of the system for proper segmentation. Case h012 is an image obtained from a patient with the papilloma virus, which creates sacs that are valid portions of the airways. A rendering and cross section are shown in Figure 12. Since the sacs occupy a substantial volume, the explosion parameter, E, was set to $70,000 \mathrm{~mm}^{3}$. Morphology-based methods such as the hybrid method tend to fail due to their inability to identify the shape and consistency of the sacs.

The hybrid algorithm considerably reduced the time required for segmentation compared with the morphology method, suiting it for clinical use. The outputs of these two methods are similar, but in addition to the speed advantage, the hybrid method has better use of larger operators. These operators are only applied to locations with airways of similar sizes. Again, the larger operators have no theoretical relevance in locations where there are no 
similarly sized airways. Hence, the hybrid method is less likely to produce as many false candidates, making reconstruction faster and the output more successful. The hybrid method was more than 20 times faster than the morphology method for labeling and about $8 \%$ faster for reconstruction.

These results indicate that adaptive 3D region growing is appropriate when a fast segmentation is needed for path planning. The hybrid approach offers better edge localization at the cost of more computation time. Both methods are necessary for clinical use, however. Additionally, given the choice of reconstruction kernel, the $\mathrm{A}$ and $\mathrm{B}$ kernels tend to produce better results.

Although human intervention is required to select prefiltering of the data, the use of the median filter before segmentation increases the robustness of all three algorithms. Adaptive region growing benefits the most from prefiltering, since region growing is prone to parenchymal leakage. The median filter helps to reduce the number of connections between airways and the parenchyma.

The system described here is being used as part of a VB analysis system to guide path selection and treatment planning $(11,15)$. The ability to segment the airways offers a basis for variety of image analysis methods. Path calculations, rendering, and quantitative analysis all depend on accurate airway segmentation.

\section{ACKNOWLEDGMENTS}

The authors thank Jered Sieren for help with the image acquisition and Janice Cook-Granroth, BS, and Angela Delsing for help with the image processing.

\section{REFERENCES}

1. Hoffman EA, McLennan G. Assessment of the pulmonary structurefunction relationship and clinical outcomes measures: quantitative volumetric CT of the lung. Acad Radiol 1997; 4:758-776.

2. Kazerooni EA. High-resolution CT of the lungs. AJR Am J Roentgenol 2001; 177:501-519.

3. Rubin GD, Beaulieu CF, Argiro V, et al. Perspective volume rendering of CT and MR images: applications for endoscopic imaging. Radiology 1996; 199:321-330.

4. Summers RM, Feng DH, Holland SM, Sneller MC, Shelhamer JH. Virtual bronchoscopy: segmentation method for real-time display. Radiology 1996; 200:857-862.

5. Summers RM. Image gallery: a tool for rapid endobronchial lesion detection and display using virtual bronchoscopy. J Digit Imaging 1998; 11(suppl 1):53-55.

6. Englmeier $\mathrm{KH}$, Haubner M, Kraplicher C, et al. Virtual bronchoscopy based on spiral-CT images. Proc SPIE 1999; 3335:427-438.

7. Konin E, Katz M, Rozenman J, Ben-Shlush A, Itzchak Y, Szeinberg A. Virtual bronchoscopy in children: early clinical experience. AJR Am J Roentgenol 1998; 171:1699-1702.
8. Higgins WE, Ramaswamy K, Swift R, McLennan G, Hoffman EA. Virtual bronchoscopy for 3D pulmonary image assessment: state of the art and future needs. RadioGraphics 1998; 18:761-778.

9. Haponik EF, Aquino SL, Vining DJ. Virtual bronchoscopy. Clin Chest Med 1999; 20:201-217.

10. Aquino SL, Vining DJ. Virtual bronchoscopy. Clin Chest Med 1999; 20:725-730.

11. Sherbondy AJ, Kiraly AP, Austin AL, et al. Virtual bronchoscopic approach for combining 3D CT and endoscopic video. Proc SPIE 2000; 3978:104-116.

12. Mori K, Hasegawa J, Suenaga Y, Toriwaki J. Automated anatomical labeling of the bronchial branch and its application to the virtual bronchoscopy system. IEEE Trans Med Imaging 2000; 19:103-114.

13. Higgins WE, Helferty JP, Hoffman EA, Kiraly AP, Ross AF, McLennan G. In vivo validation of CT-guided bronchoscopic biopsy (abstr). Am J Respir Crit Care Med 2001; 16:A776.

14. McLennan G, Helferty JP, Hoffman EA, Sherbondy AJ, Ferguson JS, Higgins WE. Virtual endoscopic guidance of bronchoscopy: phantom studies (abstr). Am J Respir Crit Care Med 2001; 16:A777.

15. Higgins WE, Kiraly AP, Sherbondy AJ, Turlington JZ, Hoffman EA, McLennan G. Use of integrated virtual-bronchoscopic analysis during live bronchoscopy (abstr). Radiology 2001; 221(P):719.

16. Amirav I, Kramer SS, Grunstein MM, Hoffman EA. Assessment of methacholine-induced airway constriction by ultrafast high-resolution computed tomography. J Appl Physiol 1993; 75:2239-2250.

17. Pisupati C, Wolff L, Mitzner W, Zerhouni E. Tracking 3-D pulmonary tree structure. In: Proceedings of the IEEE Workshop on Math Meth in Biomed Image Anal. Piscataway, NJ: IEEE Press, 1996; 160-169.

18. Reinhardt JM, D'Souza ND, Hoffman EA. Accurate measurement of intrathoracic airways. IEEE Trans Med Imaging 1997; 16:820-827.

19. Sauret V, Goatman KA, Fleming JS, Bailey AG. Semi-automated tabulation of the 3D topology and morphology of branching networks using CT: application to the airway tree. Phys Med Biol 1999; 44:16251638.

20. Swift RD, Kiraly AP, Sherbondy AJ, et al. Automatic axis generation for virtual bronchoscopic assessment of major airway obstructions. Comput Med Imaging Graph 2002; 26:103-118.

21. Sonka M, Park W, Hoffman EA. Rule-based detection of intrathoracic airway trees. IEEE Trans Med Imaging 1996; 15:314-326.

22. Bilgen $D$. Segmentation and analysis of the human airway tree from 3D x-ray CT images. Thesis. University of lowa, lowa City, 2000.

23. Sonka M, Park W, Hoffman E. Validation of an enhanced knowledgebased method for segmentation and quantitative analysis of intrathoracic airway trees from three-dimensional CT images. Proc SPIE 1995; 2433:158-166.

24. Wood S, Zerhouni E, Hoford J, Hoffman E, Mitzner W. Measurement of three-dimensional lung tree structures by using computed tomography. J Appl Physiol 1995; 79:1687-1697.

25. Mori K, Hasegawa J, Toriwaki J, Anno H, Katada K. Recognition of bronchus in three dimensional x-ray CT images with application to virtualized bronchoscopy system. In: Proceedings of the 13th International Conference on Pattern Recognition. Vol 3. Piscataway, NJ: IEEE Press, 1996; 528-532.

26. Pisupati C, Wolff L, Mitzner W, Zerhouni E. Segmentation of 3D pulmonary trees using mathematical morphology. In: Mathematical morphology and its applications to image and signal processing. Atlanta, Ga: Kluwer Academic Publishers, 1996; 409-416.

27. Swift RD, Higgins WE, Hoffman EA, McLennan G, Reinhardt JM. Automatic axis generation for 3D virtual-bronchoscopic assessment. Proc SPIE 1998; 3337:73-84.

28. Prêteux F, Fetita C, Grenier P, Capderou A. Modeling, segmentation and caliber estimation of bronchi in high-resolution computerized tomography. J Electron Imaging 1999; 8:36-45.

29. Fetita C, Prêteux F. Bronchial tree modeling and 3D reconstruction. Proc SPIE 2000; 4121:16-29.

30. Law TY, Heng PA. Automated extraction of bronchus from 3D CT images of lung based on genetic algorithm and 3D region growing. Proc SPIE 2000; 3979:906-916. 
31. Kiraly AP, Higgins WE, Hoffman EA, McLennan G, Reinhardt J. 3D human airway segmentation for virtual bronchoscopy. Proc SPIE 2002; 4683:16-29.

32. Choi HS, Haynor DR, Kim Y. Partial volume tissue classification of multichannel magnetic resonance images: a mixel model. IEEE Trans Med Imaging 1991; 10:395-407.

33. Soltanian-Zadeh H, Windham JP, Yagle AE. Optimal transformation for correcting partial volume averaging effects in magnetic resonance imaging. IEEE Trans Med Imaging 1993; 40:1204-1212.

34. Santago P, Gage HD. Statistical models of partial volume effect. IEEE Trans Image Proc 1995; 4:1531-1540.

35. Tardif PM. Virtual reality in radiology: virtual intervention. Proc SPIE 2001; 4322:1005-1014.

36. Kalender W. Computed tomography: fundamentals, system technology, image quality, applications. Munich, Germany: Publicis MCD Verlag, 2000.

37. Park W, Hoffman EA, Sonka M. Segmentation of intrathoracic airway trees: a fuzzy logic approach. IEEE Trans Med Imaging 1998; 17:489_ 497.

38. Schlatholter T, Lorenz C, Carlsen IC, Renisch S, Deschamps T. Simultaneous segmentation and tree reconstruction of the airways for virtual bronchoscopy. Proc SPIE 2002; 4684.
39. Serra J. Image analysis and mathematical morphology. San Diego, Calif: Academic Press, 1988.

40. Gonzalez RC, Woods RE. Digital image processing. Reading, Mass: Addison Wesley, 1992.

41. Hu S, Hoffman EA, Reinhardt JM. Automatic lung segmentation for accurate quantitation of volumetric x-ray CT images. IEEE Trans Med Imaging 2001; 20:490-498.

42. Kiraly AP, Higgins WE. $3 D$ human airway segmentation for virtual bronchoscopy. In: IEEE Int Conference on Image Processing. Piscataway, NJ: IEEE Press, 2002.

43. Lorensen WE, Cline HE. Marching cubes: a high resolution 3D surface construction algorithm. Comput Graph 1987; 21:163-169.

44. Helferty JP, Sherbondy AJ, Hoffman EA, McLennan G, Higgins WE. Experiments in virtual-endoscopy guidance of bronchoscopy. Proc SPIE 2001; 4321:111-121.

45. Helferty JP, Higgins WE. Technique for registering 3D virtual CT images to endoscopic video. In: IEEE Int Conf Image Processing. Piscataway, NJ: IEEE Press, 2001; 893-896.

46. Schroeder W, Martin K, Lorensen B. The visualization toolkit: an object-oriented approach to 3D graphics. Upper Saddle River, NJ: Prentice Hall, 1997.

47. Turlington JZ, Higgins WE. New techniques for efficient sliding thinslab volume visualization. IEEE Trans Med Imaging 2001; 20:823-835. 